\title{
Mitochondrial Trafficking in Neurons
}

\author{
Thomas L. Schwarz \\ F.M. Kirby Neurobiology Center, Children's Hospital Boston, and Department of Neurobiology, Harvard \\ Medical School, Boston, Massachusetts 02115 \\ Correspondence: Thomas.schwarz@childrens.harvard.edu
}

Neurons, perhaps more than any other cell type, depend on mitochondrial trafficking for their survival. Recent studies have elucidated a motor/adaptor complex on the mitochondrial surface that is shared between neurons and other animal cells. In addition to kinesin and dynein, this complex contains the proteins Miro (also called RhoT1/2) and milton (also called TRAK1/2) and is responsible for much, although not necessarily all, mitochondrial movement. Elucidation of the complex has permitted inroads for understanding how this movement is regulated by a variety of intracellular signals, although many mysteries remain. Regulating mitochondrial movement can match energy demand to energy supply throughout the extraordinary architecture of these cells and can control the clearance and replenishing of mitochondria in the periphery. Because the extended axons of neurons contain uniformly polarized microtubules, they have been useful for studying mitochondrial motility in conjunction with biochemical assays in many cell types.

\section{THE IMPORTANCE OF MITOCHONDRIAL MOVEMENT TO NEURONS}

ive imaging of mitochondria has transformed our understanding of these organelles from static lumps afloat in a cytoplasmic soup to animated actors that slide and fuse and divide (Jakobs 2006). Their dance is captivating, even when we are uncertain of its purpose. In neurons, and especially in their axons and dendrites, the trafficking of mitochondria is essential and perhaps more orderly than in other cells. Long-range intracellular transport in animal cells is accomplished primarily by microtubule-based motors-kinesins and dynein — and this is true also for the movement of mitochondria (Ligon and Steward 2000b; Hollenbeck and Saxton 2005). Axons contain linear arrays of uniformly polarized microtubules, with the mi- nus ends in the cell body and the plus ends in the distal tips. This uniform polarity has made neurons particularly useful for studying transport. Moreover, in neuronal cultures, the axons lie flat and are typically about a micrometer in diameter. The mitochondria therefore move within an easily visualized plane and, as long as the cell body or growth cone of the axon can be identified, it is easy to distinguish plus-end from minus-end directed movement and to follow mitochondria for $100 \mu \mathrm{m}$ or more. Moreover, whereas mitochondria in many cell types form a complex reticulum, axonal mitochondria have separated from the reticulum and exist as discrete organelles of typically $1-3 \mu \mathrm{m}$ in length; for unknown reasons, those in dendrites tend to be longer than those in axons (Chang et al. 2006). In some preparations, shorter mitochon-

Editors: Douglas C. Wallace and Richard J. Youle

Additional Perspectives on Mitochondria available at www.cshperspectives.org

Copyright (C) 2013 Cold Spring Harbor Laboratory Press; all rights reserved; doi: 10.1101/cshperspect.a011304

Cite this article as Cold Spring Harb Perspect Biol 2013;5:a011304 
dria are more frequently in motion than longer ones (Misgeld et al. 2007), but the relationship of size to motility is not yet clear.

The urgency of proper mitochondrial trafficking in neurons arises from their exceptional cellular morphology. Whereas most cells are measured in micrometers or tens of micrometers, neurons extend their axons and dendrites for millimeters, centimeters, and, in the case of human peripheral nerves or corticospinal tracts, up to a meter. Thus the neuron poses an extreme case for mitochondrial distribution and the need to supply energy to far-flung cellular regions. This challenge is probably why mutations of motor proteins found in all cell types give rise to selectively neurological pathologies in humans (Baloh 2007; De Vos et al. 2008); the stress on the axonal transport system is such that perturbations that another cell could survive cause degeneration in neurons.

Indeed, the regions of the neuron that have the highest demand for mitochondrial ATP production, that is, the highest energy consumption, are the synapses, and these specializations are often located at the extremities of the cell. When a single synaptic vesicle releases a transmitter at a synapse, it opens tens to hundreds of ion channels on the postsynaptic membrane, which allows up to 70 million ions to enter the cell at that point. This influx will require comparable millions of ATP to be hydrolyzed to pump the ions back across the plasma membrane. With hundreds or thousands of synapses impinging on a cell, it is easy to see why a recent imaging study determined that a resting cortical neuron consumes 4.7 billion ATP molecules per second (Zhu et al. 2012). It is not only the synapses that consume energy; action potentials also require ATP to restore ion gradients, axonal transport is energy intensive, and, of course, there are all the "normal" requirements of a cell. Thus, achieving the correct distribution of mitochondria may be a particularly challenging biological problem for the cell. It is not a question of simply recognizing the polarity of the cell and distinguishing an axon from a dendrite, but rather of distributing mitochondria to all regions so as to match their density to the local demand for ATP, $\mathrm{Ca}^{2+}$ buffering, and other mitochondrial functions. The task is further complicated by changes in energy use that require changes in mitochondrial distribution. An interesting illustration of this phenomenon is the behavior of mitochondria in a myelinated nerve, a phenomenon recently analyzed in detail (Ohno et al. 2011). Most mitochondria in myelinated nerves reside in stationary pools in the internode and juxtaparanode. Electrical activity, however, will slow or arrest the movement of mitochondria as they traverse the node of Ranvier and a blockade of electrical activity increases the motile fraction. These modulations of movement probably represent a response to the change in energy supply necessitated by the opening of ion channels at the nodes when action potentials are firing.

Nor is the traffic of mitochondria a one-time event to populate the periphery. These postmitotic cells need to survive for the lifetime of the organism, although the lifetime of mitochondrial proteins is likely to be a matter of weeks. This means there must be constant turnover of mitochondria throughout the cell, including the clearance of older, damaged components and the delivery of new materials, most of which are encoded by nuclear genes. Three processes are likely to contribute to this maintenance. The first is the constant movement of a population of mitochondria in axons and dendrites. Determinations of the fraction moving at any given moment vary, but typically fall in the range of $10 \%-$ $40 \%$ in motion, with $60 \%-90 \%$ stationary (for examples, see Morris and Hollenbeck 1993; Ligon and Steward 2000a; Misgeld et al. 2007; Russo et al. 2009; Wang and Schwarz 2009). Of those in motion, roughly half are moving anterograde (away from the cell body) and half retrograde (toward the cell body). This movement likely reflects a major mechanism for the delivery and exchange of newly synthesized mitochondrial components. One topic of ongoing debate is whether the anterograde-moving population comprises healthy, refreshed mitochondria and those moving retrograde represent older, damaged mitochondria with lower mitochondrial membrane potentials, as determined from voltage-sensing dyes. An early study found that the membrane potential was indeed diminished in 
retrogradely moving mitochondria (Miller and Sheetz 2004), but a subsequent study saw no difference (Verburg and Hollenbeck 2008). But whether or not these populations differ, the fact that there is such extensive movement means that there is opportunity for mitochondrial proteins synthesized in the soma to be delivered to the periphery.

The second important factor for maintaining peripheral mitochondria is mitochondrial fission and fusion. Because these processes are observed in axons and dendrites, as they are in all cells, they allow the exchange of materials between mitochondria (Amiri and Hollenbeck 2008). Even brief moments of contact can involve fusion and the extensive exchange of proteins in each compartment of the mitochondrion, as has been shown in non-neuronal cells (Liu et al. 2009). Therefore, the mitochondria that appear to be stationary, at least for the duration of what can be successfully imaged, may be refreshed in place by exchanging proteins with the motile fraction. Mitochondrial fission and fusion are important to the viability of neuronal mitochondria; mutations in mitofusin give rise to a peripheral neuropathy (Zuchner et al. 2004; Kijima et al. 2005) and cause progressive decreases in the integrity of mitochondrial DNA and membrane potential (Chen et al. 2007). Naturally, if there were no mitochondrial movement, fusions would be unlikely to occur and indeed, moving mitochondria have a higher rate of fusion than stationary mitochondria (Liu et al. 2009) and the mechanisms of movement and fusion may be mechanistically coupled (Misko et al. 2010).

The third factor is the possibility of local biogenesis of mitochondria in addition to what occurs in the cell body. Although the extent to which local synthesis contributes to the mitochondrial pool is unknown (much certainly occurs in the soma [Davis and Clayton 1996]), there are two strong lines of evidence that it occurs. In an elegant experiment, Amiri and Hollenbeck (2008) explanted sympathetic ganglia and, after axons had grown out from the ganglion, excised the cell bodies and observed BrdU incorporation into the mitochondrial DNA of the severed axons. In addition to DNA replication, local synthesis of mitochondrial proteins is also likely; several studies have found transcripts for local synthesis of mitochondrial proteins (Gioio et al. 2001; Aschrafi et al. 2010; Yoon et al. 2012). Thus local biogenesis of mitochondria may assist in maintaining healthy mitochondria in axons and dendrites. Moreover, local biogenesis would have the potential to respond rapidly to local changes brought about by either mitochondrial damage or increased demand. At present, it is unknown whether the nucleus is informed about the state of the mitochondrial supply in the neuronal periphery, what such a signal would be, or how quickly it could resupply a particular region in the neuronal arbor.

\section{A KEY MOTOR/ADAPTOR COMPLEX FOR MITOCHONDRIAL MOTILITY}

Recently, a motor/adaptor complex was described that mediates the transport of mitochondria in neurons and possibly most animal cells (Fig. 1) (Stowers et al. 2002; Fransson et al. 2003, 2006; Brickley et al. 2005; Guo et al. 2005; Glater et al. 2006; Brickley and Stephenson 2011). The core of this complex consists of three proteins: the heavy chain of the conventional kinesin-1, a protein anchored to the outer sur-

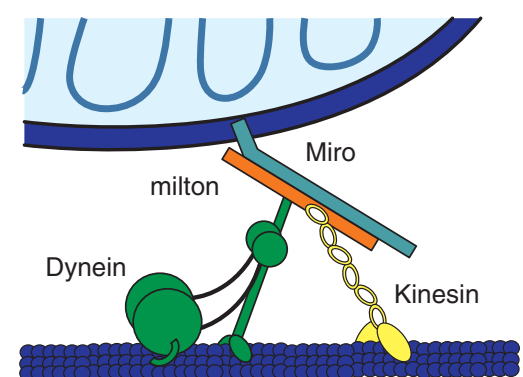

Figure 1. The mitochondrial motor/adaptor complex. The anterograde motor Kinesin-1 (also called kinesin heavy chain or Kif5) and the retrograde motor (the dynein/dynactin complex) are able to carry many cellular cargoes but are bound to mitochondria by their interactions with two mitochondrion-specific proteins: milton and Miro. Miro is carboxy-terminal anchored to the outer membrane of the mitochondrion. Milton serves as an adaptor between Miro and the motors. (Figure courtesy of Jarom Chung.) 
face of the mitochondrion called Miro (also known as RhoT1 and RhoT2), and milton (also known as TRAK1 and TRAK2), a protein that links kinesin and Miro. Dynein, as will be discussed below, interacts with milton and Miro. The stoichiometry of the complex is not yet known, but the properties of the individual proteins and their relevance to mitochondrial transport have been elucidated through genetic, biochemical, and imaging studies.

The kinesin heavy chain (KHC) or kinesin1 motor has three isoforms in mammals: Kif5A, $\mathrm{B}$, and $\mathrm{C}$. All have an amino-terminal motor domain that moves toward the plus end of the microtubule and a large coiled-coil tail that mediates its dimerization. Cargo binding occurs via the carboxy-terminal portion (reviewed in Vale 2003). The significance of KHC for mitochondrial movement is evident from the phenotypes of mutations in mouse (Tanaka et al. 1998) and Drosophila (Hurd and Saxton 1996) and from its association with the other proteins of the motor/adaptor complex (Stowers et al. 2002; Brickley et al. 2005; Glater et al. 2006).

The adaptor protein milton was named for the blind seventeenth century poet, John Milton, because it was identified in a Drosophila mutant screen for blind flies (Stowers et al. 2002). The significance of milton for mitochondrial transport was established by the phenotype of these mutants. In photoreceptors homozygous for the mutation, axons and terminals were completely devoid of mitochondria, although functional and morphologically normal mitochondria persisted in the somata of these cells (Stowers et al. 2002; Gorska-Andrzejak et al. 2003). Moreover, milton cofractionated with mitochondria, coprecipitated with KHC, and could cause the redistribution of mitochondria when overexpressed in COS cells (Stowers et al. 2002; Glater et al. 2006). The two mammalian isoforms of milton are called TRAK1 and TRAK2 (trafficking protein kinesin-binding) and sometimes GRIF1 and OIP106 for historical reasons, as discussed below (Beck et al. 2002; Iyer et al. 2003; Brickley et al. 2005), but for simplicity's sake will be discussed in aggregate as milton. Milton lacks an obvious domain structure other than an extensive coiled-coil region, which includes a significant region of homology with Huntingtin-associated protein 1 (HAP-1) (Stowers et al. 2002), a protein also implicated in organelle traffic (Gauthier et al. 2004). The ability of milton to associate with mitochondria when expressed in cells, to alter their distribution, and to bind $\mathrm{KHC}$ is shared by the fly and mammalian homologs (Stowers et al. 2002; Brickley et al. 2005, 2011; Glater et al. 2006; Smith et al. 2006). The interaction of TRAK1 with KHC is direct (Smith et al. 2006) and uses the cargo-binding region of $\mathrm{KHC}$ near the carboxyl terminus. Conventionally, the KHC binds cargo via the kinesin light chain (KLC), but milton is in competition with KLC for binding to this region of KHC (Glater et al. 2006). The role of KLC in mitochondrial trafficking is therefore unclear. Although it has been reported to be present in mitochondrial fractions (Khodjakov et al. 1998), it is not detected in the milton/Miro/KHC complex and is dispensable for mitochondrial transport in Drosophila photoreceptors (Glater et al. 2006) and cultured mammalian epithelial cells (Trejo et al. 2010). It seems likely that, for mitochondria, milton will replace KLC as the linker between cargo and motor.

Miro (Fig. 2) serves as the membrane anchor that links milton, and thereby kinesin, to the mitochondrial surface (Glater et al. 2006). Miro has two domains of homology with small GTPases and those domains are separated by a linker with two EF-hand motifs. At the carboxyl terminus there is a trans-membrane domain that tethers it to the outer surface of the outer

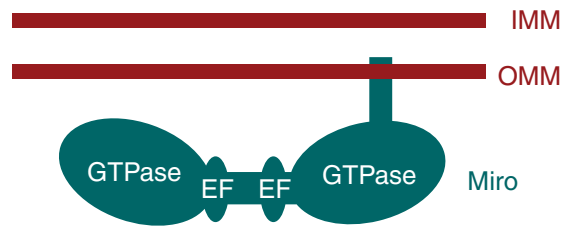

Figure 2. Domain structure of Miro (RhoT1/2). Miro has two GTPase domains that are distantly related to the small GTPases of the ras superfamily. These domains are separated by a pair of $\mathrm{Ca}^{2+}$-binding $\mathrm{EF}$ hands. At the carboxyl terminus of the protein, a short trans-membrane domain anchors Miro in the outer mitochondrial membrane (OMM). 
mitochondrial membrane (Fransson et al. 2003, 2006). There is a single fly Miro (Guo et al. 2005), but two mammalian isoforms that are sometimes called RhoT1 and RhoT2 (Aspenstrom et al. 2007). A more distant homolog in yeast, GEM1p, is necessary for proper mitochondrial morphology and inheritance (Frederick et al. 2004). Thus, the importance of the protein for regulating mitochondrial distribution appears to be very ancient, even though yeast does not use kinesin and microtubules for mitochondrial movement (Frederick and Shaw 2007). The importance of Miro for mitochondrial movement was hinted at by changes in mitochondrial distribution in overexpression studies in cells (Fransson et al. 2003), but established more directly by two parallel approaches. The first, a Drosophila mutant screen very similar to that which isolated milton alleles, isolated alleles of miro and found that they, too, lacked axonal and synaptic mitochondria (Guo et al. 2005). The untransported mitochondria formed unusual aggregates in the neuronal somata. In parallel, biochemical studies identified milton and Miro as direct binding partners (Fransson et al. 2006; Glater et al. 2006) and found that a truncated Miro lacking its membrane anchor could serve as a dominant negative construct that prevented milton from associating with mitochondria (Glater et al. 2006).

Biochemical data originally linked milton and Miro only to kinesin, but Drosophila phenotypes indicated that they may also be needed for dynein-mediated transport. The polarity of microtubules in Drosophila dendrites is the opposite of that in axons; dynein delivers cargo into dendrites and kinesins mediate movement back to the soma. In milton mutant flies, however, the neuropil regions of the central nervous system, although $\sim 50 \%$ composed of dendrites, are lacking in mitochondria (Stowers et al. 2002), and their absence indicates that dyneindependent movement of mitochondria was also disrupted. In addition, close examination of residual mitochondria in miro mutant axons indicated that both anterograde and retrograde movement was severely impaired (Guo et al. 2005). Recently, direct evidence for a biochemical interaction of the retrograde motor with the
milton/Miro complex has been obtained (van Spronsen et al. 2013).

The abundant evidence linking milton and Miro to mitochondrial traffic does not preclude their involvement in the movement of other cargo and there are some indications that other cargos exist. Mammalian milton, for example, was also identified as a GABA receptor interacting factor (and consequently also referred to as GRIF-1) in a yeast two-hybrid screen (Beck et al. 2002); but in vivo evidence that it is required for GABA receptor transport is presently lacking. It has also been reported to interact with the endosomal protein Hrs (Kirk et al. 2006) and with a $\mathrm{K}^{+}$channel (Grishin et al. 2006).

\section{OTHER MECHANISMS FOR MITOCHONDRIAL MOVEMENT}

Is the milton/Miro/kinesin-heavy-chain complex the only mechanism by which mitochondria are transported? On the one hand, the phenotypes of Drosophila null alleles of milton and miro are extreme. In the cell types examined, mitochondria were absent from axons and dendrites. This finding, however, does not preclude the involvement of other motors and adaptors in other species, other cell types, or even within the cell bodies of neurons and there is some evidence for additional transport mechanisms. These include alternative kinesin-based mechanisms. $\mathrm{Kif} 1 \mathrm{~B} \alpha$, for example, is a Kinesin-3 motor and was reported to associate with mitochondria and to be capable of transporting them in an in vitro assay (Nangaku et al. 1994), but in vivo evidence for their involvement remains scant and null alleles of the Drosophila homolog, immaculate connections, has normal transport of mitochondria (Pack-Chung et al. 2007). This family of motors is, however, critical for the transport of synaptic vesicle precursors (Hall and Hedgecock 1991; Yonekawa et al. 1998; Pack-Chung et al. 2007; Barkus et al. 2008). A distantly related member of the Kinesin-3 family, kinesin-like protein 6 (KLP6), was found to alter mitochondrial shape in Caenorhabditis elegans and mitochondrial motility in HeLa cells and a neural cell line (Tanaka et al. 2011) when mutated or knocked down with RNA interfer- 
ence (RNAi). Whereas loss of the conventional kinesin heavy chain prevented mitochondria from entering axons at all, mutant forms of KLP6 and Kif1B $\alpha$ altered the velocity of axonal mitochondria (Tanaka et al. 2011). Both of these Kinesin-3 motors have been shown to bind to a candidate adaptor protein, the kinesin binding protein (KBP). Loss of KBP can alter the distribution of mitochondria in cultured cells (Wozniak et al. 2005) and in zebrafish (Lyons et al. 2008), although the phenotype in the latter indicated that the mitochondrial phenotype was likely to be secondary to disruption of the microtubule cytoskeleton. Three additional binding partners for $\mathrm{KHC}$ bear mentioning also: FEZ1, syntabulin, and Ran-binding protein 2 (Cai et al. 2005; Cho et al. 2007; Fujita et al. 2007; Ikuta et al. 2007). Interfering with the function of each of these can also alter mitochondrial distribution, although the mechanism of that effect is unclear. The function of these additional kinesins and binding partners, therefore, and their relationship to the milton/ Miro/KHC complex remain an intriguing issue.

In addition to microtubule-based motility, actin-based mechanisms may have a role in distributing mitochondria (Morris and Hollenbeck 1993). In yeast, this is the primary mode of mitochondrial motility (Frederick and Shaw 2007), and in neurons it may mediate movement in regions where microtubules are interrupted or absent. A study of locust photoreceptors found that mitochondria moved toward and away from the phototransduction apparatus of the cell in a light-dependent manner. This movement was prevented by disruption of the actin cytoskeleton (Sturmer et al. 1995). More recently, Myo19 was shown to be a mitochondria-associated myosin and its overexpression in a neuronal cell line increased mitochondrial motility, whereas expression of a dominant-negative tail construct decreased their movement by $40 \%$ (Quintero et al. 2009). In contrast, Myosin V and Myosin VI may have the opposite role, resisting microtubule-based mechanisms for mitochondrial movement (Pathak et al. 2010). In cultured Drosophila neurons, RNAi knockdown of these proteins increased net movement and run lengths. The function of the myosins in these cells may be to remove mitochondria from microtubules and potentially tether them to the actin cytoskeleton to create a stationary pool (Pathak et al. 2010). How the myosins bind to mitochondria and the mechanisms by which they influence mitochondrial movement, however, remain unknown.

\section{REGULATION OF MITOCHONDRIAL MOVEMENT: THE STATIONARY POOL}

Movement alone cannot achieve the correct distribution of mitochondria within the neuronal arbor-mitochondrial movement must be closely regulated. Elucidating the machinery that moves mitochondria has begun to yield insights into how that movement is regulated, but many fundamental questions remain. One of the most striking features of mitochondria in axons is that they generally fall into three classes: those that move primarily anterograde $(\sim 15 \%)$, those that move primarily retrograde $(\sim 15 \%)$, and those that appear to be stationary for an extended period $(\sim 70 \%)$. What establishes these three categories or determines when a mitochondrion will switch its behavior remains largely mysterious. One possible mechanism would be that motors bind and unbind from the surface of the organelle and thereby determine whether and how it moves. At present, this mechanism seems unlikely. Neurons transfected with fluorescently tagged KHC, together with milton and Miro, revealed that KHC was present, at least under these circumstances, on the entire population of axonal mitochondria and at roughly equal levels, whether they were stationary, moving anterograde, or moving in the dynein-driven retrograde direction (Wang and Schwarz 2009). But some indications of how these populations are determined have been found. The above-mentioned studies of myosin Vand VI in Drosophila, for example, suggest that tethering to the actin cytoskeleton may be one means of halting microtubule-based transport and creating a stationary pool (Pathak et al. 2010) (Fig. 3A).

Another likely anchoring mechanism may predominate in species that, unlike Drosophila (Goldstein and Gunawardena 2000), contain 
A Anchor to actin cytoskeleton (e.g., Myosin V)

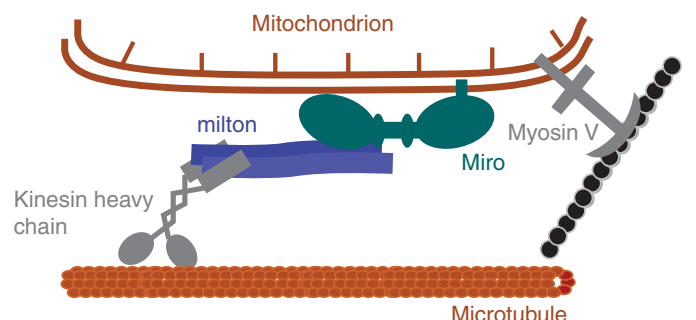

C Reversible disengagement from microtubules $\left(\mathrm{Ca}^{2+}\right)$

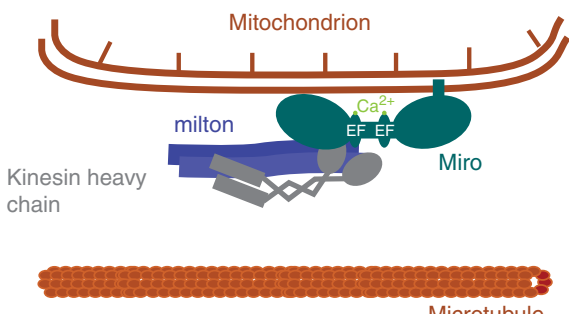

B Anchor to microtubule cytoskeleton (e.g., Syntaphilin)

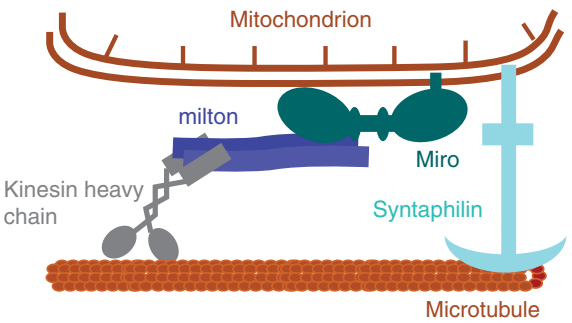

D Irreversible removal of complex from mitochondrion (e.g., PINK1/Parkin)

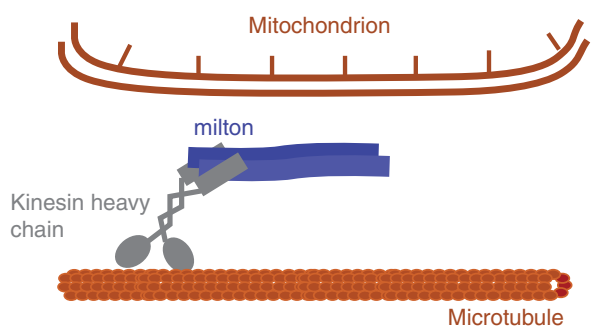

Figure 3. Four ways to stop a mitochondrion. (A) Myosin on mitochondria can inhibit their transport by tethering mitochondria to the axonal actin cytoskeleton (Pathak et al. 2010). (B) Syntaphilin is present on stationary axonal mitochondria and anchors them through interactions with microtubules (Kang et al. 2008). (C) $\mathrm{Ca}^{2+}$ binding to Miro causes a rearrangement of the complex such that the motor domain of kinesin directly binds to Miro and is blocked from binding to microtubules (Wang and Schwarz 2009). When cytosolic $\mathrm{Ca}^{2+}$ is lowered, this rearrangement can be reversed to permit continued kinesin-powered movement. (D) The PINK1/ Parkin pathway causes an irreversible dissociation of the motors from the mitochondrial surface by causing Miro to be degraded. Miro degradation by the proteasome is triggered by PINK1 phosphorylation of Miro and Parkin ubiquitinylation of Miro (Wang et al. 2011).

neurofilaments. Mitochondria have been seen by electron microscopy to be associated with neurofilaments in frozen, deep-etched axonal tissue (Hirokawa 1982). This interaction may be mediated by the direct binding to mitochondria of the phosphorylated side arms of the neurofilament heavy chain (Wagner et al. 2003). Indeed, a substantial literature associates mitochondria with intermediate filament proteins in many vertebrate cell types (e.g., Milner et al. 2000; Tao et al. 2009; Nekrasova et al. 2011). An important factor in this association may be the intermediate-filament binding protein plectin (Reipert et al. 1999; Rezniczek et al. 2003; Winter et al. 2008).

One of the most intriguing anchoring mechanisms that have been proposed involves the association of mitochondria with microtubules via the protein syntaphilin (Kang et al. 2008) (Fig. 3B). Syntaphilin is anchored in the outer membrane of the mitochondrion and also contains a microtubule-binding domain. Syntaphilin-GFP was observed preferentially on stationary mitochondria, but not if the microtubulebinding region was deleted. The dynein light chain LC8 also appears to be involved with syntaphilin-based docking (Chen et al. 2009). Mice lacking syntaphilin were found to have substantially more mitochondria in the mobile fraction (Kang et al. 2008). However, although most mitochondria are stationary in both axons and dendrites, syntaphilin is exclusively axonal (Kang et al. 2008). Moreover, syntaphilin is absent from the genome of Drosophila, although flies have a stationary pool similar to mammals. Thus, although several potential mechanisms 
for creating a stationary pool have been reported, the relative importance of each may vary and their interplay is still unclear.

\section{REGULATING MITOCHONDRIAL MOVEMENT: CYTOSOLIC Ca ${ }^{2+}$}

Cytosolic $\mathrm{Ca}^{2+}$ is one of the best-studied regulators of mitochondrial movement. Elevation of cytosolic $\mathrm{Ca}^{2+}$ stops both the anterograde and retrograde movement of mitochondria in neurons and in many cell lines (Rintoul et al. 2003; Yi et al. 2004; Chang et al. 2006; Szabadkai et al. 2006). From simultaneous measurements of free $\mathrm{Ca}^{2+}$ levels and mitochondrial dynamics, it has been determined that $50 \%$ reductions in movement will occur at concentrations of approximately $400 \mathrm{nM} \mathrm{Ca}^{2+}$ and complete arrest in the low micromolar range in $\mathrm{H} 9 \mathrm{c} 2$ cells ( $\mathrm{Yi}$ et al. 2004; Saotome et al. 2008). Elevations in $\mathrm{Ca}^{2+}$ can arise from a variety of physiological stimuli, including activation of glutamate receptors in dendrites, action potentials in axons, and neuromodulators that trigger $\mathrm{Ca}^{2+}$ release from intracellular stores (Rintoul et al. 2003; Yi et al. 2004; Saotome et al. 2008; Ohno et al. 2011). The purpose of this regulation is not firmly established, but is widely believed to be a homeostatic response to local $\mathrm{Ca}^{2+}$ influx: if passing mitochondria are arrested in areas where there is high cytosolic $\mathrm{Ca}^{2+}$, they will be able to provide both $\mathrm{Ca}^{2+}$ buffering capacity and ATP for the active transport of $\mathrm{Ca}^{2+}$ from the cell. In addition, subcellular areas with inadequate cytosolic ATP are likely to have local elevations of $\mathrm{Ca}^{2+}$, and the arrest of mitochondria in such areas will correct the lack of ATP (Yang and Steele 2000; Wang and Schwarz 2009). Tests of these possibilities have recently been made possible by manipulations of the motor/adaptor complex that selectively remove the ability of $\mathrm{Ca}^{2+}$ to regulate mitochondrial movement. Under these circumstances, mitochondrial will no longer stop at activated synapses (Macaskill et al. 2009) and neurons become more susceptible to excitotoxic cell death (Wang and Schwarz 2009), presumably because they can no longer cope with the $\mathrm{Ca}^{2+}$ that entered when glutamate receptors were activated.
The mechanism underlying this regulation has been examined in some detail. The presence of EF hands in Miro suggested a likely locus for the $\mathrm{Ca}^{2+}$-binding site and these EF hands could be disrupted by the substitution of lysine residues for two key glutamates (Miro ${ }^{\mathrm{KK}}$ [Fransson et al. 2006]). Overexpression in neurons of Miro $^{\mathrm{KK}}$ had little effect on the behavior of mitochondria in conditions of resting $\mathrm{Ca}^{2+}$, but potently disrupted the ability of elevated $\mathrm{Ca}^{2+}$ to arrest their movement (Saotome et al. 2008; Macaskill et al. 2009; Wang and Schwarz 2009). A surprise from these studies was that both the kinesin-dependent anterograde movement and the dynein-dependent retrograde movement lost their regulation by $\mathrm{Ca}^{2+}$ when Miro was mutated. Biochemical evidence has now confirmed that the dynein motor indeed interacts with the milton/Miro complex (van Spronsen et al. 2013).

How then does $\mathrm{Ca}^{2+}$ binding to the $\mathrm{EF}$ hands of Miro stop the kinesin-mediated motility of the mitochondrion? The anterograde movement is driven by the interaction of the head domain of KHC with microtubules, but Miro binds to milton and milton binds to the opposite end of KHC. A possible mechanism would involve the $\mathrm{Ca}^{2+}$ triggering the dissociation of the complex by a conformational change (Macaskill et al. 2009) that would uncouple the kinesin from the mitochondrial surface. This could either be a disruption of the $\mathrm{KHC} /$ milton interaction or the milton/Miro interaction. Though this may occur under some circumstances, there is substantial evidence against such a model: the three proteins continue to coprecipitate as a complex even in the presence of very high $\mathrm{Ca}^{2+}(2 \mathrm{mM})$; isolated mitochondria washed in high $\mathrm{Ca}^{2+}$ do not lose KHC from their surface; and in living neurons transfected with fluorescently tagged KHC, equivalent amounts of $\mathrm{KHC}$ are present on mitochondria before and after their arrest by elevated $\mathrm{Ca}^{2+}$ (Wang and Schwarz 2009). The latter experiment contained another surprise: KHC was present on all mitochondria, including those that were in the stationary pool or moving retrograde, in addition to those that moved anterograde. Apparently, the choice of direction and 
whether or not to be stationary is not governed by the association and dissociation of the motor. An alternative mechanism for $\mathrm{Ca}^{2+}$-dependent arrest has been proposed (Fig. 3C) (Wang and Schwarz 2009) in which the kinesin switches between an active state that interacts with the microtubules and an inhibited state where the motor "head" domain of kinesin binds to the $\mathrm{Ca}^{2+}$ bound state of Miro and is thereby prevented from interacting with the microtubules. The isolated $\mathrm{KHC}$ head domain will indeed bind to wild-type Miro, but only when $\mathrm{Ca}^{2+}$ is present, and not to Miro ${ }^{\mathrm{KK}}$, and this interaction prevents the head domain from cosedimenting with microtubules (Wang and Schwarz 2009). Two aspects of this mechanism are noteworthy. The first is that it is readily reversible and will allow movement to begin again as soon as $\mathrm{Ca}^{2+}$ is unbound from the EF hands. The second is that it explains the selective arrest of mitochondrial motility because the regulation by $\mathrm{Ca}^{2+}$ is not intrinsic to the motor domain, but instead is based on the interaction of that domain with the mitochondrion-specific adaptor Miro.

\section{REGULATING MITOCHONDRIAL MOVEMENT: PINK1 AND PARKIN}

Recent studies have elucidated an additional means of arresting mitochondria-one that is very different from the readily reversible arrest by $\mathrm{Ca}^{2+}$. This mechanism involves two genes, PINK1 and Parkin, which are intensively studied because mutations in them are responsible for hereditary forms of Parkinsonism (Kitada et al. 1998; Valente et al. 2004). PINK1 (PTEN-induced putative kinase 1 ) is a protein kinase and Parkin is an E3 ubiquitin ligase, and together they form a pathway that initiates the autophagic clearance of mitochondria with poor membrane potentials (reviewed elsewhere and see Whitworth and Pallanck 2009; Youle and Narendra 2011; Narendra et al. 2012). In brief, PINK1 is normally inactivated by import into mitochondria and subsequent proteolytic cleavage. When mitochondria are damaged or poisoned and do not have a sufficient membrane potential, PINK1 instead accumulates on the mitochondrial surface and causes the recruitment of Parkin. In a manner still poorly understood, ubiquitination of proteins on the mitochondrial surface initiates mitophagy. Whether as a prelude to mitophagy or perhaps just as an independent action, the PINK1/Parkin pathway also regulates mitochondrial morphology (Greene et al. 2003; Clark et al. 2006; Park et al. 2006; Yang et al. 2006; Poole et al. 2008) and this is mediated, at least in part, by targeting mitofusin for degradation (Poole et al. 2010; Tanaka et al. 2010; Chan et al. 2011). PINK1 is only effective in bringing about these changes when Parkin is present, but in some cases Parkin overexpression can alter mitochondria even in the absence of PINK1 (Clark et al. 2006; Yang et al. 2006), as might be expected in a pathway where PINK1 is upstream of Parkin.

The potential involvement of mitochondrial movement in this pathway was first indicated by the observation of a biochemical association between PINK1 and the Miro/milton complex (Weihofen et al. 2009). It was subsequently found that Parkin can also bind to this complex and that, although this association may be scant in healthy cells, it can be greatly increased if mitochondria are depolarized with carbonyl cyanide $m$-chlorophenylhydrazone (CCCP) (Wang et al. 2011). PINK1 can phosphorylate Miro on several sites, at least one of which, S156, is important for the subsequent stimulation of the Parkin function and mitochondrial arrest (Wang et al. 2011). Miro is also a substrate of Parkin (Liu et al. 2012). The consequence of activating this PINK1/Parkin pathway is the proteasome-dependent degradation of Miro and the consequent release of kinesin and milton from the mitochondrial surface (Chan et al. 2011; Wang et al. 2011) (Fig. 3D). The loss of Miro explains why overexpression of PINK1 or Parkin stops mitochondrial motion in both mammalian and Drosophila neurons and, as in the case of mitophagy, PINK1 acts upstream of Parkin and in a manner that completely depends on Parkin activity (Wang et al. 2011). Although mitochondrial movement is normal in Parkin ${ }^{-1-}$ mice, there is more movement in Drosophila axons expressing either PINK1 or Parkin RNAi relative to controls. Thus, whereas mammalian neurons may use this pathway only 
under conditions of mitochondrial damage, in Drosophila it may be part of the ongoing control of mitochondrial dynamics. It is also perhaps noteworthy that Miro and milton bind to mitofusin (Misko et al. 2010), and thus the degradation of these key regulators of mitochondrial dynamics may be coordinated.

As noted above, the association of PINK1 and Parkin with Miro is stimulated by depolarization of the mitochondrion. Mitochondrial depolarization also arrests mitochondrial transport in axons and this arrest is accompanied by the recruitment of cytosolic Parkin to the mitochondrion. Moreover, the degradation of Miro proceeds before the wholesale loss of mitochondrial proteins by mitophagy (Wang et al. 2011). It is therefore likely, although as yet undemonstrated, that halting mitochondria in some manner facilitates their clearance by mitophagy. One attractive model is that the degradation of Miro and mitofusin are both important as an early step to quarantine damaged mitochondria and prevent them from fusing with other healthy mitochondria, thereby mixing their damaged components in with the healthy population. Moving mitochondria are much more likely to fuse than stationary mitochondria (Liu et al. 2009) and therefore, together with the degradation of mitofusin, the loss of Miro will produce a population of stationary, fragmented mitochondria that are ready for engulfment by autophagosomes. Because it is a step toward clearance of the organelle, it is appropriate that the mechanism of mitochondrial arrest is by proteolysis and therefore not readily reversible.

\section{REGULATING MITOCHONDRIAL MOVEMENT: OTHER FACTORS}

The $\mathrm{Ca}^{2+}$ and PINK1/Parkin pathways are very far from the totality of regulatory influences, although for many others mechanistic information remains scant. One of the most intriguing is the influence of the nerve growh factor (NGF), which can cause a local accumulation of mitochondria where an NGF-coupled bead makes contact with a sensory axon (Chada and Hollenbeck 2003, 2004). Mitochondria that enter the portion of the axon closest to the bead tend to stop in their transit and thereby account for the accumulation. NGF activation of TrkA receptors is responsible for this effect and the pathway appears to involve PI3 kinase and the actin cytoskeleton. In light of the evidence mentioned above, that myosin interactions with actin may derail mitochondria from microtubule tracks and thereby anchor them (Pathak et al. 2010), it is possible that the NGF is increasing this set of cytoskeletal interactions.

A potentially related pathway that regulates mitochondrial motility is activated by the growth factor lysophosphatidic acid (LPA) and mediated by the small GTPase RhoA (Minin et al. 2006). Either LPA or a constitutively active RhoA will inhibit mitochondrial movement in cultured cells, including a neuron-like Drosophila cell line. RhoA can regulate interactions with the actin cytoskeleton through at least two pathways. RhoA, via Rho-kinase, can activate myosin motors (Kimura et al. 1996) and thereby potentially remove mitochondria from their microtubule tracks. In addition, RhoA can activate the actin-regulatory formin-like protein mDia (diaphanous in the fly) (Watanabe et al. 1997). The latter pathway is both necessary for and sufficient to explain the inhibitory effects of RhoA and LPA and entails the anchoring of mitochondria to the actin cytoskeleton (Minin et al. 2006). Despite the apparent importance of the actin cytoskeleton for these mechanisms of mitochondrial anchoring, the intermediate filament protein vimentin can also interact with mitochondria and influence their movement (Nekrasova et al. 2011).

Other proteins that associate with the milton/Miro complex may be significant regulators. These include HUMMR, a protein whose expression is induced by hypoxic conditions and that biases mitochondrial movement in the anterograde direction by an unknown means ( $\mathrm{Li}$ et al. 2009), and O-GlcNAc transferase (OGT), an enzyme that catalyzes the transfer of single $N$ acetylglucosamine sugars to serine and threonine residues (Iyer et al. 2003). Although this enzyme typically has only low-affinity interactions with its many substrates, it binds strongly to milton, which is also a substrate (Iyer et al. 2003). The association of OGT and milton is 
conserved in Drosophila (Glater et al. 2006) and, by an unknown mechanism, OGT expression can alter the distribution of mitochondria in cultured cells (Brickley et al. 2011). Both HUMMR, as a sensor of hypoxic stress, and OGT, as a likely sensor of metabolic state (Lazarus et al. 2009), may help mitochondria redistribute themselves in response to changing environmental and energetic conditions. In this regard, they resemble the $\mathrm{Ca}^{2+}$-sensing mechanism described above that can arrest mitochondria in regions of high-energy demand. A parallel mechanism for controlling the distribution of mitochondria entails the ability of ADP to decrease mitochondrial motility (Mironov 2007, 2009). Because ADP levels will be high where ATP consumption is high, the arrest of mitochondria by elevated ADP will cause ATP-producing mitochondria to concentrate where they are most needed.

The most recent addition to the list of motility regulators are the proteins of the Armcx family, including Alex3 (Lopez-Domenech et al. 2012). This family, characterized by multiple armadillo-like repeats, is unique to the placental mammals, localizes to mitochondria, and when overexpressed in cultured cells, including neurons, causes the mitochondria to aggregate near the nucleus. Alex3 binds to the Miro/milton complex and, when overexpressed, substantially decreases axonal transport of mitochondria (Lopez-Domenech et al. 2012). Also, as previously mentioned, mitofusins bind to the Miro/ milton complex. This association could allow Miro to regulate mitochondrial fusion or allow mitofusin to regulate mitochondrial motility and, indeed, knockdown of the mitofusin 2 expression decreased mitochondrial motility in axons (Misko et al. 2010). Mitochondrial movement and fusion are likely to be coordinated processes.

Avery different form of regulation is likely to arise from the selection of the particular isoforms of milton and Miro that are found in a cell or on a subset of mitochondria. In mammals, there are two genes apiece for both milton and Miro (often called TRAK1 and TRAK2 and RhoT1 and RhoT2). This diversity is augmented by alternative splicing. Both Drosophila and mammalian milton are found in multiple splice isoforms. Although there is no functional information at present on the significance of the mammalian splice variants, the splicing of Drosophila milton appears to be highly significant. In particular, of four known forms of the aminoterminal portion, one, milton- $\mathrm{C}$, does not bind to kinesin heavy chain when expressed in cultured cells and does not recruit $\mathrm{KHC}$ to mitochondria, although it contains the KHC-binding sequence (Glater et al. 2006). This amino terminus, therefore, may be a regulatory domain that can influence the association and dissociation of the motor from the complex in vivo. Selective loss of splice isoforms in a Drosophila allele also suggested isoform-specific functions (Cox and Spradling 2006). The two mammalian genes, TRAK1 and TRAK2, encode proteins that similarly differ in their interactions with the motors: TRAK1 will bind both kinesin and dynein, but TRAK2 preferentially interacts with dynein (van Spronsen et al. 2013).

Other regulatory signals may impinge on the motors themselves or on the microtubules and their associated proteins. These include microtubule-affinity regulating kinases (MARKs) (Mandelkow et al. 2004), JNK1 (Morfini et al. 2006, 2009), histone deacetylase 6 (Chen et al. 2010), and cyclin-dependent kinases (Ratner et al. 1998; Morfini et al. 2004; Holzbaur 2010). These influences, however, are unlikely to be selective for mitochondrial motility but rather will influence axonal transport broadly.

\section{SUMMARY: DISTRIBUTING MITOCHONDRIA, COMPLEX SOLUTIONS TO A COMPLEX PROBLEM}

Given the complexity of the task facing the neuron-distributing mitochondria over long distances and matching their distribution to an ever-changing demand for energy-perhaps it is not surprising that such a rich and complex regulatory machinery has evolved. This apparatus needs to contend not only with the delivery of healthy mitochondria to the appropriate regions, but also to their retention in areas with a high requirement for their $\mathrm{Ca}^{2+}$-buffering capacity and energy production. What has 


\section{T.L. Schwarz}

emerged so far is a core apparatus of milton and Miro that will link the kinesin and dynein motors to the mitochondrial surface and an exceptional number of signaling pathways and regulatory proteins that will interact with that core and with the other cytoskeletal elements, particularly the actin network, which can stabilize mitochondria. When this machinery is compromised there are pathological consequences that may include the impaired ability of neurons to survive excitotoxic stimuli or to be cleared from cells when damaged. There is much still to decipher. The stoichiometry of the motor/adaptor complex is unknown and these proteins cry out for greater structural information. The significance of distinct isoforms and splice variants remains unclear and many modulatory pathways still lack a detailed mechanistic understanding. Most pressingly perhaps, the in vivo relevance of the regulatory pathways remains unclear. Nevertheless, studies of motility in animal cells have uncovered a remarkably complex and dynamic life for the mitochondrion.

\section{REFERENCES}

${ }^{*}$ Reference is also in this collection.

Amiri M, Hollenbeck PJ. 2008. Mitochondrial biogenesis in the axons of vertebrate peripheral neurons. Dev Neurobiol 68: $1348-1361$.

Aschrafi A, Natera-Naranjo O, Gioio AE, Kaplan BB. 2010 Regulation of axonal trafficking of cytochrome $c$ oxidase IV mRNA. Mol Cell Neurosci 43: 422-430.

Aspenstrom P, Ruusala A, Pacholsky D. 2007. Taking Rho GTPases to the next level: The cellular functions of atypical Rho GTPases. Exp Cell Res 313: 3673-3679.

Baloh RH. 2007. Mitochondrial dynamics and peripheral neuropathy. Neuroscientist 14: 12-18.

Barkus RV, Klyachko O, Horiuchi D, Dickson BJ, Saxton WM. 2008. Identification of an axonal kinesin-3 motor for fast anterograde vesicle transport that facilitates retrograde transport of neuropeptides. Mol Biol Cell 19: 274-283.

Beck M, Brickley K, Wilkinson HL, Sharma S, Smith M, Chazot PL, Pollard S, Stephenson FA. 2002. Identification, molecular cloning, and characterization of a nove GABAA receptor-associated protein, GRIF-1. J Biol Chem 277: 30079-30090.

Brickley K, Stephenson FA. 2011. Trafficking kinesin protein (TRAK)-mediated transport of mitochondria in axons of hippocampal neurons. J Biol Chem 286: 1807918092.

Brickley K, Smith MJ, Beck M, Stephenson FA. 2005. GRIF1 and OIP106, members of a novel gene family of coiled- coil domain proteins: Association in vivo and in vitro with kinesin. J Biol Chem 280: 14723-14732.

Brickley K, Pozo K, Stephenson FA. 2011. N-acetylglucosamine transferase is an integral component of a kinesindirected mitochondrial trafficking complex. Biochim Biophys Acta 1813: 269-281.

Cai Q, Gerwin C, Sheng ZH. 2005. Syntabulin-mediated anterograde transport of mitochondria along neuronal processes. J Cell Biol 170: 959-969.

Chada SR, Hollenbeck PJ. 2003. Mitochondrial movement and positioning in axons: The role of growth factor signaling. J Exp Biol 206: 1985-1992.

Chada SR, Hollenbeck PJ. 2004. Nerve growth factor signaling regulates motility and docking of axonal mitochondria. Curr Biol 14: 1272-1276.

Chan NC, Salazar AM, Pham AH, Sweredoski MJ, Kolawa NJ, Graham RL, Hess S, Chan DC. 2011. Broad activation of the ubiquitin-proteasome system by Parkin is critical for mitophagy. Hum Mol Genet 20: 1726-1737.

Chang DT, Honick AS, Reynolds IJ. 2006. Mitochondrial trafficking to synapses in cultured primary cortical neurons. J Neurosci 26: 7035-7045.

Chen H, McCaffery JM, Chan DC. 2007. Mitochondrial fusion protects against neurodegeneration in the cerebellum. Cell 130: 548-562.

Chen YM, Gerwin C, Sheng ZH. 2009. Dynein light chain LC8 regulates syntaphilin-mediated mitochondrial docking in axons. J Neurosci 29: 9429-9438.

Chen S, Owens GC, Makarenkova H, Edelman DB. 2010. HDAC6 regulates mitochondrial transport in hippocampal neurons. PLoS ONE 5: e10848.

Cho KI, Cai Y, Yi H, Yeh A, Aslanukov A, Ferreira PA. 2007. Association of the kinesin-binding domain of RanBP2 to KIF5B and KIF5C determines mitochondria localization and function. Traffic 8: 1722-1735.

Clark IE, Dodson MW, Jiang C, Cao JH, Huh JR, Seol JH, Yoo SJ, Hay BA, Guo M. 2006. Drosophila pink1 is required for mitochondrial function and interacts genetically with parkin. Nature 441: 1162-1166.

Cox RT, Spradling AC. 2006. Milton controls the early acquisition of mitochondria by Drosophila oocytes. Development 133: 3371-3377.

Davis AF, Clayton DA. 1996. In situ localization of mitochondrial DNA replication in intact mammalian cells. $J$ Cell Biol 135: 883-893.

De Vos KJ, Grierson AJ, Ackerley S, Miller CC. 2008. Role of axonal transport in neurodegenerative diseases. Annu Rev Neurosci 31: 151-173.

Fransson A, Ruusala A, Aspenstrom P. 2003. Atypical Rho GTPases have roles in mitochondrial homeostasis and apoptosis. J Biol Chem 278: 6495-6502.

Fransson S, Ruusala A, Aspenstrom P. 2006. The atypical Rho GTPases Miro-1 and Miro-2 have essential roles in mitochondrial trafficking. Biochem Biophys Res Commun 344: $500-510$.

Frederick RL, Shaw JM. 2007. Moving mitochondria: Establishing distribution of an essential organelle. Traffic 8: 1668-1675.

Frederick RL, McCaffery JM, Cunningham KW, Okamoto K, Shaw JM. 2004. Yeast Miro GTPase, Gem1p, regulates 
mitochondrial morphology via a novel pathway. J Cell Biol 167: 87-98.

Fujita T, Maturana AD, Ikuta J, Hamada J, Walchli S, Suzuki T, Sawa H, Wooten MW, Okajima T, Tatematsu K, et al. 2007. Axonal guidance protein FEZ1 associates with tubulin and kinesin motor protein to transport mitochondria in neurites of NGF-stimulated PC12 cells. Biochem Biophys Res Commun 361: 605-610.

Gauthier LR, Charrin BC, Borrell-Pages M, Dompierre JP, Rangone H, Cordelieres FP, De Mey J, MacDonald ME, Lessmann V, Humbert S, et al. 2004. Huntingtin controls neurotrophic support and survival of neurons by enhancing BDNF vesicular transport along microtubules. Cell 118: 127-138.

Gioio AE, Eyman M, Zhang H, Lavina ZS, Giuditta A, Kaplan BB. 2001. Local synthesis of nuclear-encoded mitochondrial proteins in the presynaptic nerve terminal. J Neurosci Res 64: 447-453.

Glater EE, Megeath LJ, Stowers RS, Schwarz TL. 2006. Axonal transport of mitochondria requires milton to recruit kinesin heavy chain and is light chain independent. J Cell Biol 173: 545-557.

Goldstein LS, Gunawardena S. 2000. Flying through the Drosophila cytoskeletal genome. J Cell Biol 150: F63-F68.

Gorska-Andrzejak J, Stowers RS, Borycz J, Kostyleva R, Schwarz TL, Meinertzhagen IA. 2003. Mitochondria are redistributed in Drosophila photoreceptors lacking milton, a kinesin-associated protein. J Comp Neurol 463: $372-388$.

Greene JC, Whitworth AJ, Kuo I, Andrews LA, Feany MB, Pallanck LJ. 2003. Mitochondrial pathology and apoptotic muscle degeneration in Drosophila parkin mutants. Proc Natl Acad Sci 100: 4078-4083.

Grishin A, Li H, Levitan ES, Zaks-Makhina E. 2006. Identification of $\gamma$-aminobutyric acid receptor-interacting factor 1 (TRAK2) as a trafficking factor for the $\mathrm{K}^{+}$channel Kir2.1. J Biol Chem 281: 30104-30111.

Guo X, Macleod GT, Wellington A, Hu F, Panchumarthi S, Schoenfield M, Marin L, Charlton MP, Atwood HL, Zinsmaier KE. 2005. The GTPase dMiro is required for axonal transport of mitochondria to Drosophila synapses. Neuron 47: 379-393.

Hall DH, Hedgecock EM. 1991. Kinesin-related gene unc104 is required for axonal transport of synaptic vesicles in C. elegans. Cell 65: 837-847.

Hirokawa N. 1982. Cross-linker system between neurofilaments, microtubules, and membranous organelles in frog axons revealed by the quick-freeze, deep-etching method. J Cell Biol 94: 129-142.

Hollenbeck PJ, Saxton WM. 2005. The axonal transport of mitochondria. J Cell Sci 118: 5411-5419.

Holzbaur E. 2010. Axonal transport: CDKs as traffic signals for motor-ists along the axon? Curr Biol 20: R641-R642.

Hurd DD, Saxton WM. 1996. Kinesin mutations cause motor neuron disease phenotypes by disrupting fast axonal transport in Drosophila. Genetics 144: 1075-1085.

Ikuta J, Maturana A, Fujita T, Okajima T, Tatematsu K, Tanizawa K, Kuroda S. 2007. Fasciculation and elongation protein $\zeta-1$ (FEZ1) participates in the polarization of hippocampal neuron by controlling the mitochondrial motility. Biochem Biophys Res Commun 353: 127-132.
Iyer SP, Akimoto Y, Hart GW. 2003. Identification and cloning of a novel family of coiled-coil domain proteins that interact with O-GlcNAc transferase. J Biol Chem 278: 5399-5409.

Jakobs S. 2006. High resolution imaging of live mitochondria. Biochim Biophys 1763: 561-575.

Kang JS, Tian JH, Pan PY, Zald P, Li C, Deng C, Sheng ZH. 2008. Docking of axonal mitochondria by syntaphilin controls their mobility and affects short-term facilitation. Cell 132: 137-148.

Khodjakov A, Lizunova EM, Minin AA, Koonce MP, Gyoeva FK. 1998. A specific light chain of kinesin associates with mitochondria in cultured cells. Mol Biol Cell 9: 333-343.

Kijima K, Numakura C, Izumino H, Umetsu K, Nezu A, Shiiki T, Ogawa M, Ishizaki Y, Kitamura T, Shozawa Y, et al. 2005. Mitochondrial GTPase mitofusin 2 mutation in Charcot-Marie-Tooth neuropathy type 2A. Human Genet 116: 23-27.

Kimura K, Ito M, Amano M, Chihara K, Fukata Y, Nakafuku M, Yamamori B, Feng J, Nakano T, Okawa K, et al. 1996. Regulation of myosin phosphatase by Rho and Rho-associated kinase ( $\rho$-kinase). Science 273: 245-248.

Kirk E, Chin LS, Li L. 2006. GRIF1 binds Hrs and is a new regulator of endosomal trafficking. J Cell Sci 119: 46894701.

Kitada T, Asakawa S, Hattori N, Matsumine H, Yamamura Y, Minoshima S, Yokochi M, Mizuno Y, Shimizu N. 1998. Mutations in the parkin gene cause autosomal recessive juvenile parkinsonism. Nature 392: 605-608.

Lazarus BD, Love DC, Hanover JA. 2009. O-GlcNAc cycling: Implications for neurodegenerative disorders. Int $\mathrm{J}$ Biochem Cell Biol 41: 2134-2146.

Li Y, Lim S, Hoffman D, Aspenstrom P, Federoff HJ, Rempe DA. 2009. HUMMR, a hypoxia- and HIF- $1 \alpha$-inducible protein, alters mitochondrial distribution and transport. J Cell Biol 185: 1065-1081.

Ligon LA, Steward O. 2000a. Movement of mitochondria in the axons and dendrites of cultured hippocampal neurons. J Comp Neurol 427: 340-350.

Ligon LA, Steward O. 2000b. Role of microtubules and actin filaments in the movement of mitochondria in the axons and dendrites of cultured hippocampal neurons. J Comp Neurol 427: 351-361.

Liu X, Weaver D, Shirihai O, Hajnoczky G. 2009. Mitochondrial "kiss-and-run": Interplay between mitochondrial motility and fusion-fission dynamics. $E M B O J \mathbf{2 8}$ : 3074-3089.

Liu S, Sawada T, Lee S, Yu W, Silverio G, Alapatt P, Millan I, Shen A, Saxton W, Kanao T, et al. 2012. Parkinson's disease-associated kinase PINK1 regulates Miro protein level and axonal transport of mitochondria. PLoS Genet 8: e1002537.

Lopez-Domenech G, Serrat R, Mirra S, D’Aniello S, Somorjai I, Abad A, Vitureira N, Garcia-Arumi E, Alonso MT, Rodriguez-Prados M, et al. 2012. The Eutherian Armcx genes regulate mitochondrial trafficking in neurons and interact with Miro and Trak2. Nat Commun 3: 814.

Lyons DA, Naylor SG, Mercurio S, Dominguez C, Talbot WS. 2008. KBP is essential for axonal structure, outgrowth and maintenance in zebrafish, providing insight 
T.L. Schwarz

into the cellular basis of Goldberg-Shprintzen syndrome. Development 135: 599-608.

Macaskill AF, Rinholm JE, Twelvetrees AE, Arancibia-Carcamo IL, Muir J, Fransson A, Aspenstrom P, Attwell D, Kittler JT. 2009. Mirol is a calcium sensor for glutamate receptor-dependent localization of mitochondria at synapses. Neuron 61: 541-555.

Mandelkow EM, Thies E, Trinczek B, Biernat J, Mandelkow E. 2004. MARK/PAR1 kinase is a regulator of microtubule-dependent transport in axons. J Cell Biol 167: 99110.

Miller KE, Sheetz MP. 2004. Axonal mitochondrial transport and potential are correlated. J Cell Sci 117: 27912804.

Milner DJ, Mavroidis M, Weisleder N, Capetanaki Y. 2000. Desmin cytoskeleton linked to muscle mitochondrial distribution and respiratory function. J Cell Biol 150: $1283-1298$.

Minin AA, Kulik AV, Gyoeva FK, Li Y, Goshima G, Gelfand VI. 2006. Regulation of mitochondria distribution by RhoA and formins. J Cell Sci 119: 659-670.

Mironov SL. 2007. ADP regulates movements of mitochondria in neurons. Biophys J 92: 2944-2952.

Mironov SL. 2009. Complexity of mitochondrial dynamics in neurons and its control by ADP produced during synaptic activity. Int J Biochem Cell Biol 41: 2005-2014.

Misgeld T, Kerschensteiner M, Bareyre FM, Burgess RW, Lichtman JW. 2007. Imaging axonal transport of mitochondria in vivo. Nature Methods 4: 559-561.

Misko A, Jiang S, Wegorzewska I, Milbrandt J, Baloh RH 2010. Mitofusin 2 is necessary for transport of axonal mitochondria and interacts with the Miro/Milton complex. J Neurosci 30: 4232-4240.

Morfini G, Szebenyi G, Brown H, Pant HC, Pigino G, DeBoer S, Beffert U, Brady ST. 2004. A novel CDK5-dependent pathway for regulating GSK3 activity and kinesindriven motility in neurons. EMBO J 23: 2235-2245.

Morfini G, Pigino G, Szebenyi G, You Y, Pollema S, Brady ST. 2006. JNK mediates pathogenic effects of polyglutamineexpanded androgen receptor on fast axonal transport. Nat Neurosci 9: 907-916.

Morfini GA, You YM, Pollema SL, Kaminska A, Liu K, Yoshioka K, Bjorkblom B, Coffey ET, Bagnato C, Han D, et al. 2009. Pathogenic Huntingtin inhibits fast axonal transport by activating JNK3 and phosphorylating kinesin. Nat Neurosci 12: 864-871.

Morris RL, Hollenbeck PJ. 1993. The regulation of bidirectional mitochondrial transport is coordinated with axonal outgrowth. J Cell Sci 104: 917-927.

Nangaku M, Sato-Yoshitake R, Okada Y, Noda Y, Takemura R, Yamazaki H, Hirokawa N. 1994. KIF1B, a novel microtubule plus end-directed monomeric motor protein for transport of mitochondria. Cell 79: 1209-1220.

* Narendra D, Walker JE, Youle R. 2012. Mitochondrial quality control mediated by PINK1 and Parkin: Links to Parkinsonism. Cold Spring Harb Perspect Biol 4: a011338.

Nekrasova OE, Mendez MG, Chernoivanenko IS, TyurinKuzmin PA, Kuczmarski ER, Gelfand VI, Goldman RD, Minin AA. 2011. Vimentin intermediate filaments modulate the motility of mitochondria. Mol Biol Cell 22: $2282-2289$.
Ohno N, Kidd GJ, Mahad D, Kiryu-Seo S, Avishai A, Komuro H, Trapp BD. 2011. Myelination and axonal electrical activity modulate the distribution and motility of mitochondria at CNS nodes of Ranvier. J Neurosci 31: 7249-7258.

Pack-Chung E, Kurshan PT, Dickman DK, Schwarz TL. 2007. A Drosophila kinesin required for synaptic bouton formation and synaptic vesicle transport. Nat Neurosci 10: $980-989$.

Park J, Lee SB, Lee S, Kim Y, Song S, Kim S, Bae E, Kim J, Shong M, Kim JM, et al. 2006. Mitochondrial dysfunction in Drosophila PINK1 mutants is complemented by parkin. Nature 441: 1157-1161.

Pathak D, Sepp KJ, Hollenbeck PJ. 2010. Evidence that myosin activity opposes microtubule-based axonal transport of mitochondria. J Neurosci 30: 8984-8992.

Poole AC, Thomas RE, Andrews LA, McBride HM, Whitworth AJ, Pallanck LJ. 2008. The PINK1/Parkin pathway regulates mitochondrial morphology. Proc Natl Acad Sci 105: $1638-1643$.

Poole AC, Thomas RE, Yu S, Vincow ES, Pallanck L. 2010. The mitochondrial fusion-promoting factor mitofusin is a substrate of the PINK1/parkin pathway. PLOS ONE 5: e10054.

Quintero OA, DiVito MM, Adikes RC, Kortan MB, Case LB, Lier AJ, Panaretos NS, Slater SQ, Rengarajan M, Feliu M et al. 2009. Human Myo19 is a novel myosin that associates with mitochondria. Curr Biol 19: 2008-2013.

Ratner N, Bloom GS, Brady ST. 1998. A role for cyclindependent kinase(s) in the modulation of fast anterograde axonal transport: Effects defined by olomoucine and the APC tumor suppressor protein. J Neurosci 18: 7717-7726.

Reipert S, Steinbock F, Fischer I, Bittner RE, Zeold A, Wiche G. 1999. Association of mitochondria with plectin and desmin intermediate filaments in striated muscle. Exp Cell Res 252: 479-491.

Rezniczek GA, Abrahamsberg C, Fuchs P, Spazierer D, Wiche G. 2003. Plectin 5'-transcript diversity: Short alternative sequences determine stability of gene products, initiation of translation and subcellular localization of isoforms. Human Mol Genet 12: 3181-3194.

Rintoul GL, Filiano AJ, Brocard JB, Kress GJ, Reynolds IJ. 2003. Glutamate decreases mitochondrial size and movement in primary forebrain neurons. J Neurosci 23: 78817888.

Russo GJ, Louie K, Wellington A, Macleod GT, Hu F, Panchumarthi S, Zinsmaier KE. 2009. Drosophila Miro is required for both anterograde and retrograde axonal mitochondrial transport. J Neurosci 29: 5443-5455.

Saotome M, Safiulina D, Szabadkai G, Das S, Fransson A, Aspenstrom P, Rizzuto R, Hajnoczky G. 2008. Bidirectional $\mathrm{Ca}^{2+}$-dependent control of mitochondrial dynamics by the Miro GTPase. Proc Natl Acad Sci 105: 20728 20733.

Smith MJ, Pozo K, Brickley K, Stephenson FA. 2006. Mapping the GRIF-1 binding domain of the kinesin, KIF5C, substantiates a role for GRIF- 1 as an adaptor protein in the anterograde trafficking of cargoes. J Biol Chem 281: 27216-27228.

Stowers RS, Megeath LJ, Gorska-Andrzejak J, Meinertzhagen IA, Schwarz TL. 2002. Axonal transport of mitochon- 
dria to synapses depends on milton, a novel Drosophila protein. Neuron 36: 1063-1077.

Sturmer K, Baumann O, Walz B. 1995. Actin-dependent light-induced translocation of mitochondria and ER cisternae in the photoreceptor cells of the locust Schistocerca gregaria. J Cell Sci 108: 2273-2283.

Szabadkai G, Simoni AM, Bianchi K, De Stefani D, Leo S, Wieckowski MR, Rizzuto R. 2006. Mitochondrial dynamics and $\mathrm{Ca}^{2+}$ signaling. Biochim Biophys Acta 1763: $442-449$.

Tanaka Y, Kanai Y, Okada Y, Nonaka S, Takeda S, Harada A, Hirokawa N. 1998. Targeted disruption of mouse conventional kinesin heavy chain, kif5B, results in abnormal perinuclear clustering of mitochondria. Cell 93: 11471158.

Tanaka A, Cleland MM, Xu S, Narendra DP, Suen DF, Karbowski M, Youle RJ. 2010. Proteasome and p97 mediate mitophagy and degradation of mitofusins induced by Parkin. J Cell Biol 191: 1367-1380.

Tanaka K, Sugiura Y, Ichishita R, Mihara K, Oka T. 2011. KLP6: A newly identified kinesin that regulates the morphology and transport of mitochondria in neuronal cells. J Cell Sci 124: 2457-2465.

Tao GZ, Looi KS, Toivola DM, Strnad P, Zhou Q, Liao J, Wei Y, Habtezion A, Omary MB. 2009. Keratins modulate the shape and function of hepatocyte mitochondria: A mechanism for protection from apoptosis. J Cell Sci 122: 3851-3855.

Trejo HE, Lecuona E, Grillo D, Szleifer I, Nekrasova OE, Gelfand VI, Sznajder JI. 2010. Role of kinesin light chain-2 of kinesin-1 in the traffic of Na,K-ATPase-containing vesicles in alveolar epithelial cells. FASEB $J$ 24: 374-382.

Vale RD. 2003. The molecular motor toolbox for intracellular transport. Cell 112: 467-480.

Valente EM, Abou-Sleiman PM, Caputo V, Muqit MM, Harvey K, Gispert S, Ali Z, Del Turco D, Bentivoglio AR, Healy DG, et al. 2004. Hereditary early-onset Parkinson's disease caused by mutations in PINK1. Science 304: $1158-1160$.

van Spronsen M, Mikhaylova M, Lipka J, Schlager MA, van den Heuvel DJ, Kuijpers M, Wulf PS, Keijzer N, Demmers J, Kapitein LC, et al. 2013. TRAK/Milton motor-adaptor proteins steer mitochondrial trafficking to axons and dendrites. Neuron 77: 485-502.

Verburg J, Hollenbeck PJ. 2008. Mitochondrial membrane potential in axons increases with local nerve growth factor or semaphorin signaling. J Neurosci 28: 8306-8315.

Wagner OI, Lifshitz J, Janmey PA, Linden M, McIntosh TK, Leterrier JF. 2003. Mechanisms of mitochondria-neurofilament interactions. J Neurosci 23: 9046-9058.

Wang X, Schwarz TL. 2009. The mechanism of $\mathrm{Ca}^{2+}$-dependent regulation of kinesin-mediated mitochondrial motility. Cell 136: 163-174.
Wang X, Winter D, Ashrafi G, Schlehe J, Wong YL, Selkoe D, Rice S, Steen J, LaVoie MJ, Schwarz TL. 2011. PINK1 and Parkin target Miro for phosphorylation and degradation to arrest mitochondrial motility. Cell 147: 893-906.

Watanabe N, Madaule P, Reid T, Ishizaki T, Watanabe G, Kakizuka A, Saito Y, Nakao K, Jockusch BM, Narumiya S. 1997. p140mDia, a mammalian homolog of Drosophila diaphanous, is a target protein for Rho small GTPase and is a ligand for profilin. EMBO J 16: 3044-3056.

Weihofen A, Thomas KJ, Ostaszewski BL, Cookson MR, Selkoe DJ. 2009. Pink1 forms a multiprotein complex with Miro and Milton, linking Pink1 function to mitochondrial trafficking. Biochemistry 48: 2045-2052.

Whitworth AJ, Pallanck LJ. 2009. The PINK1/Parkin pathway: A mitochondrial quality control system? J Bioenerg Biomembr 41: 499-503.

Winter L, Abrahamsberg C, Wiche G. 2008. Plectin isoform $1 \mathrm{~b}$ mediates mitochondrion-intermediate filament network linkage and controls organelle shape. J Cell Biol 181: 903-911.

Wozniak MJ, Melzer M, Dorner C, Haring HU, Lammers R. 2005. The novel protein KBP regulates mitochondria localization by interaction with a kinesin-like protein. $B M C$ Cell Biol 6: 35 .

Yang Z, Steele DS. 2000. Effects of cytosolic ATP on spontaneous and triggered $\mathrm{Ca}^{2+}$-induced $\mathrm{Ca}^{2+}$ release in permeabilised rat ventricular myocytes. J Physiol 523: 29-44.

Yang Y, Gehrke S, Imai Y, Huang Z, Ouyang Y, Wang JW, Yang L, Beal MF, Vogel H, Lu B. 2006. Mitochondrial pathology and muscle and dopaminergic neuron degeneration caused by inactivation of Drosophila Pink1 is rescued by Parkin. Proc Natl Acad Sci 103: 10793-10798.

Yi M, Weaver D, Hajnoczky G. 2004. Control of mitochondrial motility and distribution by the calcium signal: A homeostatic circuit. J Cell Biol 167: 661-672.

Yonekawa Y, Harada A, Okada Y, Funakoshi T, Kanai Y, Takei Y, Terada S, Noda T, Hirokawa N. 1998. Defect in synaptic vesicle precursor transport and neuronal cell death in KIF1A motor protein-deficient mice. J Cell Biol 141: $431-441$.

Yoon BC, Jung H, Dwivedy A, O’Hare CM, Zivraj KH, Holt CE. 2012. Local translation of extranuclear lamin B promotes axon maintenance. Cell 148: 752-764.

Youle RJ, Narendra DP. 2011. Mechanisms of mitophagy. Nat Rev Mol Cell Biol 12: 9-14.

Zhu XH, Qiao H, Du F, Xiong Q, Liu X, Zhang X, Ugurbil K, Chen W. 2012. Quantitative imaging of energy expenditure in human brain. Neuroimage 60: 2107-2117.

Zuchner S, Mersiyanova IV, Muglia M, Bissar-Tadmouri N, Rochelle J, Dadali EL, Zappia M, Nelis E, Patitucci A, Senderek J, et al. 2004. Mutations in the mitochondrial GTPase mitofusin 2 cause Charcot-Marie-Tooth neuropathy type 2A. Nat Genet 36: 449-451. 


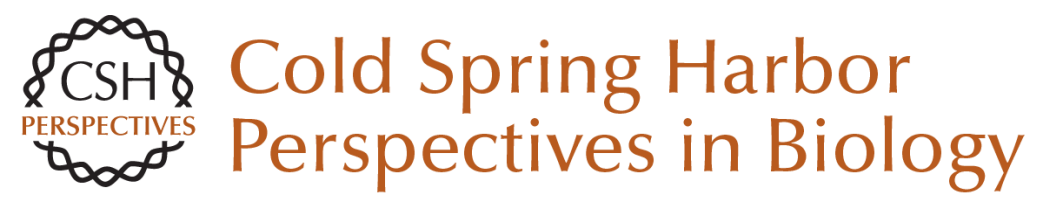

\section{Mitochondrial Trafficking in Neurons}

Thomas L. Schwarz

Cold Spring Harb Perspect Biol 2013; doi: 10.1101/cshperspect.a011304

Subject Collection Mitochondria

Altered Sulfide $\left(\mathrm{H}_{2} \mathrm{~S}\right)$ Metabolism in Ethylmalonic Encephalopathy Valeria Tiranti and Massimo Zeviani

Mitochondrial DNA Genetics and the Heteroplasmy Conundrum in Evolution and Disease

Douglas C. Wallace and Dimitra Chalkia

The Role of Mitochondria in Cellular Iron-Sulfur Protein Biogenesis: Mechanisms, Connected

Processes, and Diseases Oliver Stehling and Roland Lill

Mechanisms of Mitochondrial Fission and Fusion Alexander M. van der Bliek, Qinfang Shen and Sumihiro Kawajiri

The Mitochondrial Nucleoid: Integrating Mitochondrial DNA into Cellular Homeostasis Robert Gilkerson, Liliana Bravo, Iraselia Garcia, et al.

Relevance of Mitochondrial Genetics and Metabolism in Cancer Development Giuseppe Gasparre, Anna Maria Porcelli, Giorgio Lenaz, et al.

Mitochondrial Quality Control Mediated by PINK1 and Parkin: Links to Parkinsonism Derek Narendra, John E. Walker and Richard Youle

Mitochondrial Evolution

Michael W. Gray
Where Killers Meet--Permeabilization of the Outer Mitochondrial Membrane during Apoptosis Tom Bender and Jean-Claude Martinou

Mitochondrial Biogenesis through Activation of

Nuclear Signaling Proteins

John E. Dominy and Pere Puigserver

Mitochondrial Trafficking in Neurons

Thomas L. Schwarz

Mitochondrial Dysfunction and Defective

Autophagy in the Pathogenesis of Collagen VI

Muscular Dystrophies

Paolo Bernardi and Paolo Bonaldo

Clinical and Molecular Features of POLG-Related

Mitochondrial Disease

Jeffrey D. Stumpf, Russell P. Saneto and William C. Copeland

Mitochondrial Metabolism, Sirtuins, and Aging Michael N. Sack and Toren Finkel

Mechanisms of Protein Sorting in Mitochondria Diana Stojanovski, Maria Bohnert, Nikolaus Pfanner, et al.

For additional articles in this collection, see http://cshperspectives.cshlp.org/cgi/collection/

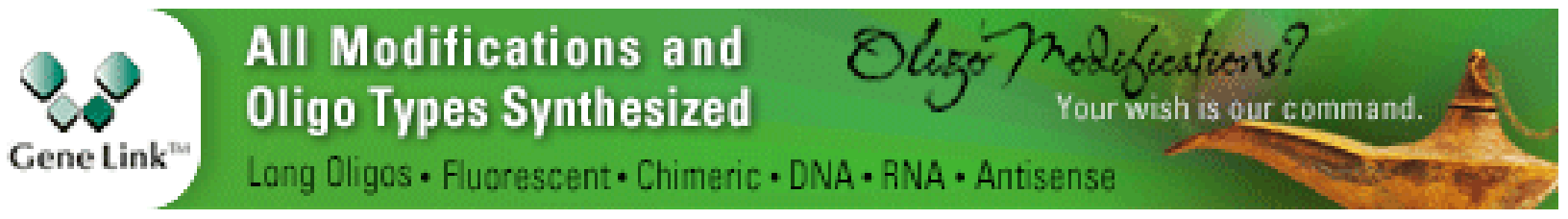

